\title{
Development of a Data Mining Driven Forecasting Software Tool for Quality Function Deployment
}

\author{
${ }^{*}$ Shivani K Purohit, ${ }^{2}$ Prof. Ashish K. Sharma \\ ${ }^{1,2}$ Department of Computer Technology, M.I.E.T, Gondia \\ *Email: shivani_purohit@rediffmail.com,ash5000@rediffmail.com
}

Received: 09 ${ }^{\text {th }}$ July 2018, Accepted: $14^{\text {th }}$ August 2018, Published: $31^{\text {st }}$ August 2018

\begin{abstract}
Recently, solicitation of Quality Function Deployment (QFD) has been extensively emerged in the product development market. Customer Requirements (CRs) and Design Requirements (DRs) are the two chief entity of the QFD process. However, variegation of marketplace directly influence CRs and DRs in making them more varying; that prompts the need of forecasting in QFD for gaining enhanced customer satisfaction. It can be attained by using Data Mining techniques of forecasting. As various forecasting techniques are available, selection of most efficient method is highly desired for evaluating out the most effective result. This paper offers an innovative software tool that precisely forecasts future values in QFD. The tool enables forecasting using several time series analysis techniques of data mining that sturdily lend a hand in carrying comparative analysis and assessing out the efficient technique. The tool is designed using VB.Net and MS-Access. Lastly, an example is shown to exhibit the workability of proposed forecasting tool.
\end{abstract}

Keywords: QFD, Data Mining, Time Series Analysis, Forecasting, Tool

\section{Introduction}

In todays' innovative world owing to the rapid enhancement of technology, attaining customer satisfaction has turned to be the most challenging job for organizations. Worldwide competitiveness in the marketplace has recently become the major apprehension of many organizations, which deliberate "continuous improvement" to deal with the readily varying development throughout the world encouraged by technological innovations and fluctuating customers' requirements (CRs) (Kahraman, Ertay \& Buyukozkan, 2004). To deal with this issue, currently corporations primarily emphasizes on the Mantra - 'communication with customers', the reason behind this is customers plays key role in rising evolving market place; also realizing requirements specified by them may entail to the prosperous product. The Quality Function Deployment (QFD) has emerged as one of the most promising planning tools to deal with customer's requirements. The QFD transforms the customer requirements (CRs') into design requirements (DRs) for a product, prioritizing these features while simultaneously forming development objectives for it (Karsak, 2004).
Customer requirements (CRs) are one of the crucial entity that plays key role in several phases of QFD. Whereas, DRs are answers to technical implementation of these CRs in an interim semantic of designers and engineers. In consequence of prompt divergence in marketplace, it is pretty clear that CRs would be additionally fluctuating. As product designing solely depends on previous requirements, CRs may be variable while designing and be different when it is already in the market. These dynamic CRs also results in their corresponding DRs. As a result, dealing these time lag problem and dynamic requirements have become thoughtprovoking task for designer and engineers. Thus, there is pressing need of forecasting in QFD. Forecasting future trends in QFD can be fruitful for organizations to deliver improved products, upgrade their effectiveness in market and elevate customer satisfaction. (Hsu, Wang \& Lin, 2007). In the contexture of a customer-centric products, a timely update of CRs may assist as an essential criterion to observe how things differ over time, moreover it comprises the organizations a higher ground to interoperate various tactics to encounter the future CRs (Bostaki \& Roghanian, 2013). Forecasting upcoming values in QFD would prove to be profitable in framing the forthcoming tactics and can be helpful in taking the anticipatory steps for the forthcoming situations. Many experts have employed various forecasting techniques in QFD for forecasting the future values.

Data mining has been recognized as popular means of forecasting. It is the process that explores all through the data to determine unidentified associations amongst the data that are fascinating to the consumer of the data (Alsultanny, 2013). The elementary perception of data mining practices the ancient values to estimate the future values. The application of data mining can be seen over numerous arenas for forecasting upcoming values such as crime forecasting, stock markets, demand forecast ,weekly weather reports, finance and etc.( Khedkar, \& Argiddi, 2013; Kalyankar, \& Alaspurkar, S. 2013; Olinsky, Schumacher, \& Quinn, 2016). Nevertheless, its employment on QFD is very tenuous and infrequently referred in the literature. Since past few years, researchers have promoted the use of data mining in QFD for forecasting (Huang, Li \& Ye, 2012; Lai, Hsu \& 
Kuo, 2012; Hsu, Chang \& Kuo, 2012; Bostaki \& Roghanian, 2013).

Data mining along with QFD are enormously massive processes and comprises of complicated computation, thus it turns out to be absolutely cumbrous when worked manually. As a consequence, the development of software for the incorporation of these processes can be expedient and assist in time saving. To this end, this study emphasizes on the development of forecasting software tool that integrates QFD with data mining techniques and generates future trend forecasts through various timeseries analysis techniques, such as time series analysis techniques, Single Exponential Smoothing (SE), Weighted Moving Average (WMA), Double Exponential Smoothing using Holt's and Winter's method (DEH\&W), Double Exponential Smoothing using Brown's Method (DEB), and Simple Moving Average (SMA). The proposed forecasting tool also assists in doing the comparative analysis of numerous time-series techniques of forecasting in order to estimate the proficient technique of forecasting. Mean Squared Error (MSE) is utilized for measuring the forecast precision. VB.net is used as front-end whereas for back-end support MS-Access is used. It has been comprehended that selection of the tools are proficient for development purpose. A practical example of Entity Resource Planning (ERP) system is deliberated for testing the accurateness and effectiveness of software tool with suppositious set of data.

\section{A Practical Example:}

This section describes the overall functionality of forecasting software tool. The study exploits data mining in QFD with purpose of recognizing future CRs and DRs for ERP software. It comprises set of QFD process of generating raw weights for CR and priority scores for DRs for the number of periods for generating the forecast for succeeding period. The techniques viz, $L R, S E$, WMA, DEH\&WDEB, and $S M A$; are employed over the data generated using QFD for recognizing the outcomes for following period. The forecasting of CRs and DRs are conducted using the rough set of data that has been analysed and verified further. For carrying out the QFD process, we have taken 52 customers, $6 \mathrm{CRs}, 7$ DRs, 3 competitors and 2 dealers.

The details of customer requirements and design requirements used for the ERP system is furnished in tables below:

\begin{tabular}{|l|l|}
\hline ID & Customer Requirements \\
\hline CR1 & Absolute expenditure of ownership \\
\hline CR2 & Functional fit of ERP system \\
\hline CR3 & User friendliness \\
\hline CR4 & Flexibility implies \\
\hline CR5 & Vendors reputation \\
\hline CR6 & Service and support quality \\
\hline
\end{tabular}

Table 1. Customer Requirements

\begin{tabular}{|l|l|}
\hline ID & Design Requirements \\
\hline DR1 & Percentage of aided requirement \\
\hline DR2 & $\begin{array}{l}\text { Percentage of aided requirement via } \\
\text { personalization }\end{array}$ \\
\hline DR3 & Customer count \\
\hline DR4 & Number of design engineers \\
\hline DR5 & General time span of operators' training \\
\hline DR6 & Vendors operating country \\
\hline DR7 & Vendors total revenue \\
\hline
\end{tabular}

Table 2. Design Requirements

Upon declaration of the data being processed, it is essential to initiate the QFD process to generate raw weights and priority score for each periods. Here, raw weights and priority scores are generated for 5 periods and the raw weights and priority scores time-series based forecasting techniques will be utilized for forecasting of the succeeding period. Raw weights and priority scores are generated using QFD process. Fig. 1 and fig. 2 shows the raw weights and priority scores for all 5 periods and these data is further utilized as an input to the succeeding phase.

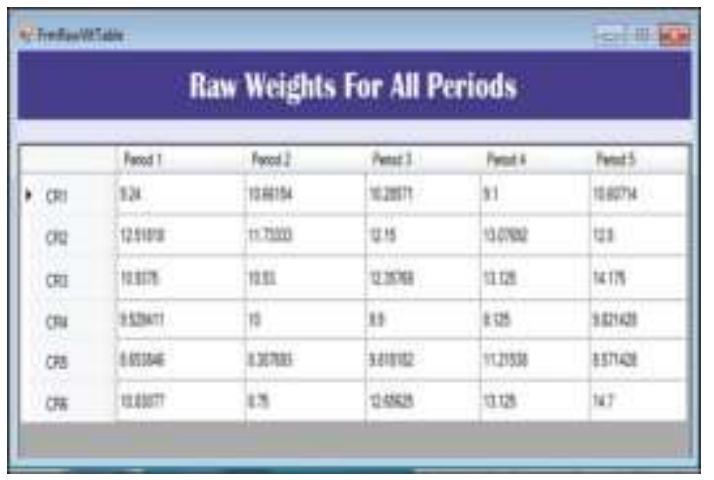

Fig. 1: Generated Raw Weights for 5 Periods

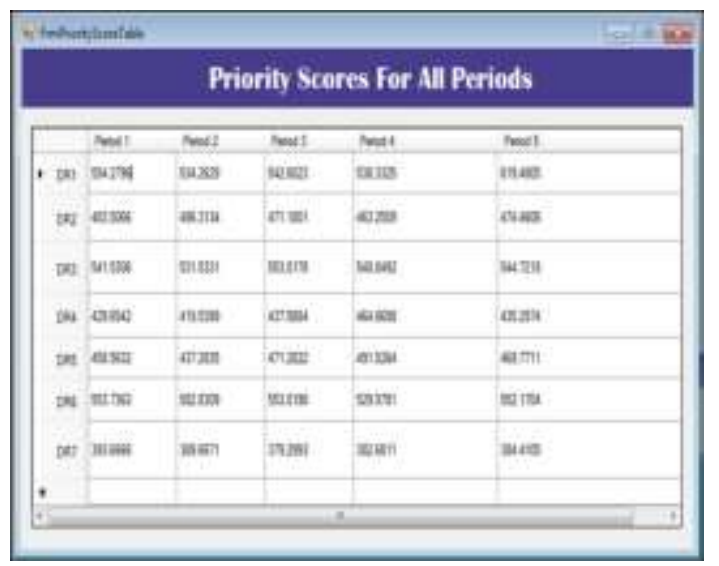

Fig. 2: Generated Priority Scores for 5 Periods Thereafter, the time-series techniques of data mining viz., $L R, S E$, WMA, $D E H \& W D E B$, and $S M A$; are consequentially employed over periodic data for generating the forecasts of CRs and DRs 
for succeeding period. Before initiating the process of forecasting, it is prerequisite to specify the some details required by these techniques. Here, we have taken 3 period SMA, 3 period WMA with weights $0.2,0.3$, and $0.5, \alpha=0.6$ for SE, $\alpha=0.4$ for DEB and $\alpha$ $=0.8 \beta=0.2$ for DEHW. These details are then exploited to generate CRs and DRs forecasting. The $\mathrm{CR}$ and DR forecasting using several forecasting techniques are depicted in Fig. 3 and fig.4 respectively.

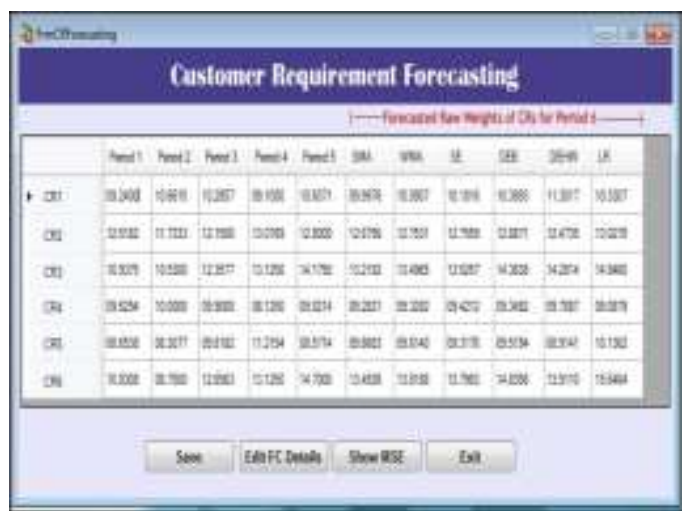

Fig. 3: Forecasted Raw Weights for Period 6

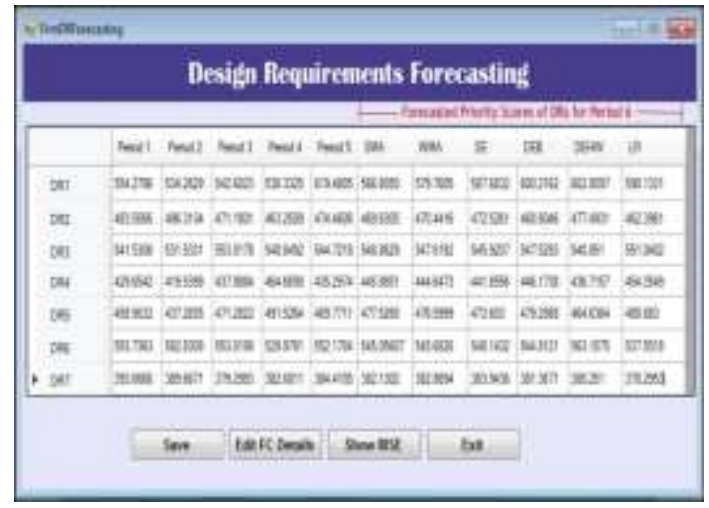

Fig. 4: Forecasted Priority Scores for Period 6

Testing and Result Analysis:

The study exploits time-series techniques of forecasting viz., $L R, S E, W M A, D E H \& W D E B$, and $S M A$ in QFD. Hence, it turns out to be immense need of evaluating out the most apt and proficient technique of determining futuristic CRs and DRs. Evaluation of forecasting techniques is based on the forecast error associated with it, to serve the purpose Mean Squared Error (MSE) is utilized here to assess the forecast accuracy. Lesser the MSE more efficient the method is. The MSE can be determined by taking the average of difference of actual value and forecasted value. The MSE of each forecasting technique for each CR and DR is obtained and depicted in the Fig. 5 and Fig. 6.

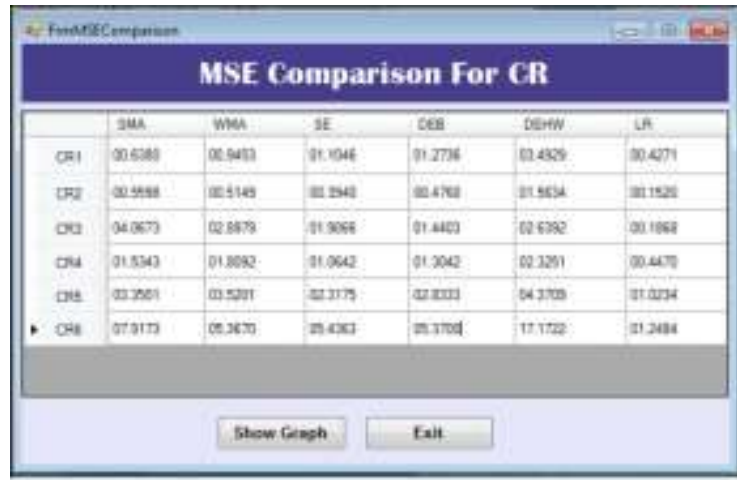

Fig. 5: MSE Comparison for CRs

\begin{tabular}{|c|c|c|c|c|c|c|}
\hline \multicolumn{6}{|c|}{ 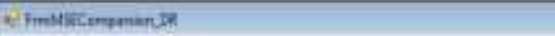 } & \multirow{2}{*}{ 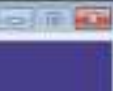 } \\
\hline \multicolumn{6}{|c|}{ MSE Comparison For DR } & \\
\hline & 5en & कास & If & cet & Detw & 내 \\
\hline ont & antsind & Porala & $16 a c m$ & $\log 29$ & Inisen & ousu \\
\hline DAI & 2ilatis & inges: & Itteal & 2452581 & seing & a.c5ises \\
\hline DR3 & 28308 & 12.2584 & $108=0$ & grux & 367596 & Elues \\
\hline oni & UAT & asimes & a) $x=1$ & Ev $25 \pi$ & incon & 17x+21 \\
\hline DFE & S46 1465 & 13inda & Ema & 203 & 105286 & $15 \mathrm{~s}$ \\
\hline \multirow{2}{*}{ 1) DAE } & $\operatorname{mos} 5 \times 20$ & s7zon & 5492197 & จ4:43 & Sue:A7 & 20979 \\
\hline & $n \times 10$ & 42020 & $\angle \cos 5$ & $4 \operatorname{sen} 4$ & Asen & 1 \\
\hline \multirow[t]{2}{*}{0} & & & $\Rightarrow$ & & & - \\
\hline & & Stow & & Fxin & & \\
\hline
\end{tabular}

Fig. 6: MSE Comparison for DRs

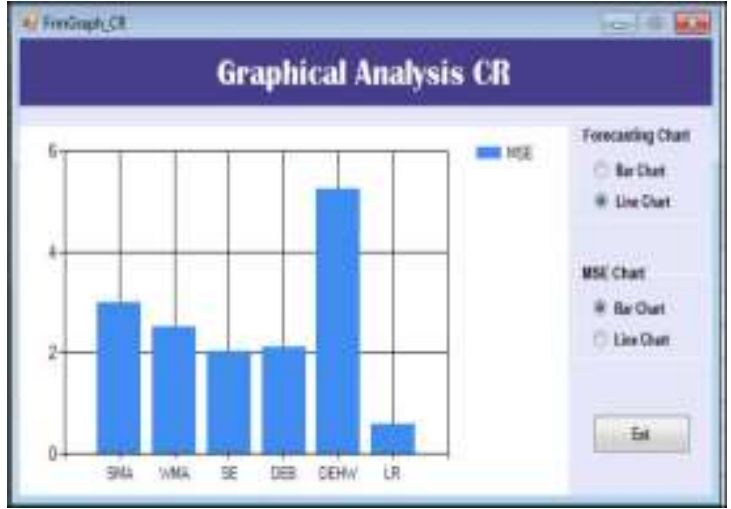

Fig 7: Graphical Analysis for CRs

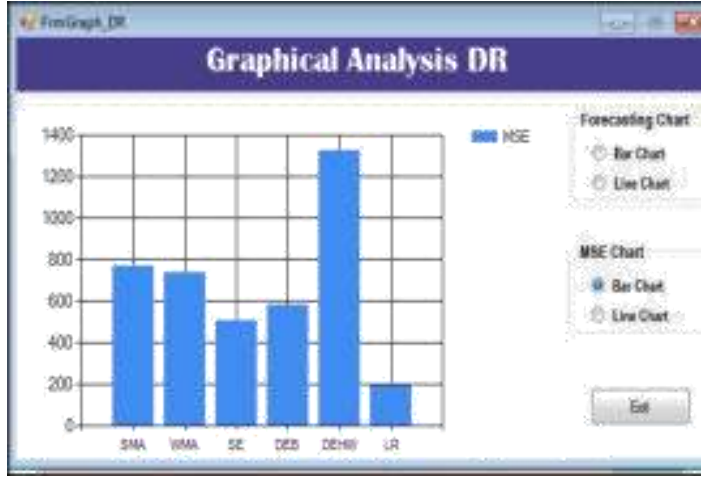

Fig 8: Graphical Analysis for DRs 
To gain the clear visualization, Fig. 7 and Fig. 8 show the graph of the MSE results. These graphs provides the apparent vision to comprehend the MSE results that can assist in analyzing the effectual forecasting technique. Bar charts are drawn out by plotting the average of MSE for each technique.

Taking CR6 as an example, it can be seen that the MSE for the CR6 using techniques SMA, WMA, SE, DEB, DEHW and LR are 07.9173, 05.3670, 05.4363, 05.4700, 17.1722 and 01.2484 respectively, as can be seen from Figure 5. It clearly depicts that the LR has least MSE as compared to other techniques. The MSE results for other CRs and DRs also have similar outcomes nearly for all consequences. Likewise, by analyzing the graphs it can be clearly recognized that the forecast error for LR is minimum. The outcomes are plotted by computing the average of MSE of all CRs and DRs for each forecasting method. The order of forecasting techniques according to their forecast accuracy is shown below:

$\mathrm{LR}>\mathrm{SE}>\mathrm{DEB}>\mathrm{WMA}>\mathrm{SMA}>\mathrm{DEHW}$

Hence, it can be comprehended that $L R$ is the most apposite method for forecasting future CRs and DRs in QFD.

\section{Conclusion}

Due to the rapid enhancement of marketplace, forecasting the future values has become pressing need of organization to deal the dynamic requirements. To serve the purpose, a unique forecasting software tool is introduced that efficiently forecasts the CRs and DRs in QFD by applying time series techniques of forecasting. This integration assists in generating the outcomes in lesser time and improves the customer satisfaction. The tool functions in three stages: first it deals with the QFD process to generate Raw weight and Priority scores, thereafter it employs the various time-series techniques such as, SMA, WMA, SE, DEB, DEH\&W, and LR on QFD data to forecast the future weights, and finally it evaluates these forecasting techniques comparatively as per their forecast accuracy. MSE is used as an evaluation criteria for identifying the apt method of forecasting. In order to test the practicability of software tool, a practical example of ERP system has been taken with data sets of 5 periods to produce forecast of succeeding period. Outcomes of study illustrates that the MSE for LR is minimum followed by SE, DEB, WMA, SMA and DEHW taking all CRs and DRs into consideration. Hence, it has been realized that the Linear Regression (LR) overtakes the other forecasting techniques and validated to be proficient technique for identifying the futuristic weights of both CRs and DRs in QFD. The result of this study can be beneficial for the designers and engineers in coping up with the futuristic CRs and DRs as well as improving the competitiveness in the global marketplace with enhanced customer satisfaction.

\section{References}

1. Kahraman, C., Ertay, T. and Buyukozkan G. 2004. A fuzzy optimization model for QFD planning process using analytic network approach. European Journal of Operational Research, Elsevier, vol. 171, no.2, pp. 390-411.

2. Karsak, E., 2004. Fuzzy multiple objective programming framework to prioritize design requirements in quality function deployment. Computers \& Industrial Engineering, Elsevier, vol. 47, pp. 149-163.

3.Hsu, H., Wang, S., and Lin. L. 2007. Using data mining to identify customer needs in quality function deployment for software design. In Proceedings of the 6th WSEAS International Conference on Artificial Intelligence, Knowledge Engineering and Data Bases, Corfu Island, Greece.

4.Bostaki, Z., and Roghanian, E. 2013. A comprehensive approach to handle the dynamics of customer's needs in quality function deployment based on linguistic variables. Decision Science Letters, Growing Science Ltd., 3, 243-258.

5. Alsultanny, Y. 2013. Labor market forecasting by using data mining. In Computer Science, International Conference on Computational Science, Elsevier. 18,1700 - 1709.

6. Khedkar, A. and Argiddi, R.V. 2013. To Study and Analyze to Foresee Market using Data Mining Technique. International Journal of Engineering Trends and Technology (IJETT), 4(9).

7. Kalyankar, M.A. and Alaspurkar, S.J. 2013. Data mining technique to analyze the metrological data. International Journal of Advanced Research in Computer Science and Software Engineering, 03(2).

8. Hebert, D.,Anderson, and Olinsky, A. and Hardin, J.M. 2014.Time series data mining: A retail application. International Journal of 
Helix Vol. 8(5): 3993- 3997

Business Analytics (IJBAN), 1(4), 51-68. doi:10.4018/ijban.2014100104.

9. Huang, H., Li, B., and Ye, Q. 201). Forecast of importance weights of customer requirements based on artificial immune system and least square support vector machine. Management Science and Engineering (ICMSE), Int. Conf.

10. Lai, J., Hsu, C., and Kuo, H. 2012. An empirical study of constructing a dynamic mining and forecasting system for the life cycle assessment-based green supply chain. WSEAS TRANSACTIONS on SYSTEMS, 11(4).

11. Hsu, H., Chang, A., and Kuo, M. 2012. Data mining QFD for the dynamic forecasting of life cycle under green supply chain. WSEAS TRANSACTIONS on COMPUTERS, 11(1).

12. Siraj, Nordin, N., \& Yusoff, N. 2008. Quality function deployment analysis based on neural network and statistical results. IJSSST, 9

(2). 